\title{
System Representations for the Zakai Class With Applications
}

\author{
Holger Boche, Senior Member, IEEE, and Ullrich J. Mönich, Student Member, IEEE
}

\begin{abstract}
The convergence behavior of a convolution representation of stable linear time-invariant (LTI) systems operating on the Zakai class of bandlimited signals is analyzed. It is shown that there are signals in the Zakai class for which the convolution integral diverges if the system is the Hilbert transform or the ideal low-pass filter with bandwidth less than or equal to the signal bandwidth. Moreover, using a previously obtained result of Habib, it is proved that the class of stable LTI systems that map the Zakai class into itself does not include the Hilbert transform and the ideal low-pass filter with bandwidth less than or equal to the signal bandwidth. Finally, it is shown that the concept of the analytical signal, which is used in communications, is problematic for the signal space $\mathcal{Z}_{\pi}$, because the operator for its computation is unbounded and discontinuous.
\end{abstract}

Index Terms - Analytical signal, bandlimited signal, convolution integral, Hilbert transform, ideal low-pass filter, system representation, Zakai class.

\section{INTRODUCTION}

A SAMPLING series or convolution integral representation of bandlimited signals is important, not only from the theoretical point of view [1]-[3], but also for practical applications. Naturally, the convergence of such representations depends on the signal space under consideration. The early results were often obtained for the space of bandlimited signals with finite energy [4]. Since then, much effort has been put in extending these results to a broader class of signals [5]-[10] and to nonuniform sampling patterns [11]-[14].

An important space is the Zakai class $\mathcal{Z}_{\pi}$, which consists of all continuous signals that satisfy

$$
\int_{-\infty}^{\infty} \frac{|f(t)|^{2}}{1+t^{2}} \mathrm{~d} t<\infty
$$

and whose Fourier transform in the distributional sense is contained in $[-\pi, \pi][6],[15]$. Mathematically, this space can be seen as a weighted $L^{2}$-space [16] with an additional band-limitation constraint. Clearly, this space is a generalization of the commonly used space of finite-energy bandlimited signals, because it also contains signals for which no statement about the concentration in the time-domain can be made. For this reason,

Manuscript received May 13, 2009; revised October 07, 2009. Date of current version July 14, 2010. This work was supported in part by the German Research Foundation (DFG) under grant BO 1734/21-1.

The authors are with the Technische Universität Berlin, Heinrich-Hertz-Chair for Mobile Communications, Einsteinufer 25, D-10578 Berlin, Germany (e-mail: holger.boche@mk.tu-berlin.de; ullrich.moenich@mk.tu-berlin.de).

Communicated by E. Serpedin, Associate Editor for Signal Processing.

Digital Object Identifier 10.1109/TIT.2010.2050824 the space $\mathcal{Z}_{\pi}$ has gained much attention, especially in the development of sampling theorems for stochastic processes [6], [15], [17], [10].

Oftentimes, the interest is not in a series or convolution integral representation of the signal itself, but in a representation of some transformation $T f$ of the signal $f$, generated by some linear time-invariant (LTI) system $T$ [18]-[20]. Then the goal is to find a system representation as a series or a convolution integral.

The problem of finding representations of stable LTI systems has been studied for a long time [21], [19]. In [19], Habib gives a convolution representation for systems operating on the Zakai space $\mathcal{Z}_{\pi}$. He proves a convolution representation of $T f$, if $f$ is in $\mathcal{Z}_{\pi}$ and $T$ is a linear system that stably maps $\mathcal{Z}_{\pi} \rightarrow \mathcal{Z}_{\pi}$ and $\mathcal{P W}_{\pi}^{2} \rightarrow \mathcal{P} \mathcal{W}_{\pi}^{2}$. This is an interesting result, because it generalizes previously obtained convolution integral representations [21] to the large signal space $\mathcal{Z}_{\pi}$.

However, in [19], no example operators that fulfill the assumptions of the theorem are given. In Section IV, we will show that the class of permissible systems, i.e., operators, is strongly restricted in [19]. For example, two important systems are not included: the ideal low-pass filter with bandwidth less than or equal to the signal bandwidth and the Hilbert transform.

In Section V, we combine our findings from Section IV with the results from [19] to show that the Hilbert transform is not a bounded operator that maps $\mathcal{Z}_{\pi}$ into $\mathcal{Z}_{\pi}$. This fact has consequences for all applications where the Hilbert transform is used. For example the computation of the analytic signal, which is a commonly used concept in signal processing and communication theory [22], [23], needs the Hilbert transform. In [23], Gabor used the analytical signal to develop a "Theory of Communication". For historical details about communication theory we would like to refer the reader to [24]. Our results show that the concept of the analytical signal is not meaningful for the Zakai class $\mathcal{Z}_{\pi}$.

\section{NOTATION AND PRELIMINARIES}

Let $\hat{f}$ denote the Fourier transform of a signal $f$, where $\hat{f}$ is to be understood in the distributional sense. $L^{p}(\mathbb{R}), 1 \leq p<\infty$, is the space of all $p$ th-power Lebesgue integrable functions on $\mathbb{R}$, with the usual norm $\|\cdot\|_{p}$, and $L^{\infty}(\mathbb{R})$ is the space of all functions for which the essential supremum norm $\|\cdot\|_{\infty}$ is finite.

For $\sigma>0$ and $1 \leq p \leq \infty$ we denote by $\mathcal{P W}_{\sigma}^{p}$ the Paley-Wiener space of signals $f$ with a representation $f(z)=$ $1 /(2 \pi) \int_{-\sigma}^{\sigma} g(\omega) \mathrm{e}^{i z \omega} \mathrm{d} \omega, z \in \mathbb{C}$, for some $g \in L^{p}(-\sigma, \sigma)$. If $f \in \mathcal{P} \mathcal{W}_{\sigma}^{p}$ then $g=\hat{f}$. The norm for $\mathcal{P} \mathcal{W}_{\sigma}^{p}, 1 \leq p<\infty$, is given by $\|f\|_{\mathcal{P} \mathcal{W}_{\sigma}^{p}}=\left(1 /(2 \pi) \int_{-\sigma}^{\sigma}|\hat{f}(\omega)|^{p} \mathrm{~d} \omega\right)^{1 / p}$. 
Furthermore, let the space $\mathcal{Z}_{\pi}$ consist of all continuous signals $f$ that satisfy $\|f\|_{\mathcal{Z}_{\pi}}:=\left(\int_{-\infty}^{\infty}|f(t)|^{2} /\left(1+t^{2}\right) \mathrm{d} t\right)^{1 / 2}<\infty$ and whose Fourier transform in the distributional sense is contained in $[-\pi, \pi]$. It is clear that $\mathcal{P} \mathcal{W}_{\pi}^{2}$ is a dense subspace of $\mathcal{Z}_{\pi}$. An important connection between compactly supported distributions and the properties of their Fourier transforms is established by the Paley-Wiener-Schwartz theorem [25].

Paley-Wiener-Schwartz Theorem: Let $f$ be a distribution supported in $[-\sigma, \sigma]$. Then $\hat{f}$ is an entire function satisfying a growth estimate

$$
|\hat{f}(z)| \leq C(1+|z|)^{N} \mathrm{e}^{\sigma|\operatorname{Im}(z)|}, \quad z \in \mathbb{C}
$$

for some $C$ and $N$. Conversely, if $F$ is an entire function satisfying the growth estimate (1) then it is the Fourier transform of a distribution with support in $[-\sigma, \sigma]$.

Before we continue the discussion, we briefly review some definitions and facts about stable LTI systems. A linear system $T: \mathcal{A} \rightarrow \mathcal{B}$, mapping signals from the space $\mathcal{A}$ to the space $\mathcal{B}$, is called stable if the operator $T$ is bounded, i.e., if $\|T\|=$ $\sup _{\|f\|_{\mathcal{A}} \leq 1}\|T f\|_{\mathcal{B}}<\infty$, and time invariant if $(T f(\cdot-a))(t)=$ $(T f)(t-a)$ for all $f \in \mathcal{A}$ and $t, a \in \mathbb{R}$.

Remark 1: Note that our definition of stability is with respect to the norms of the spaces $\mathcal{A}$ and $\mathcal{B}$, and thus is different from the concept of bounded-input bounded-output stability in general.

Mathematically, a system is an operator, i.e., a rule by which an input signal is transformed into an output signal. This operator can have different representations. For example, one possible representation for stable LTI systems operating on signals in $\mathcal{P} \mathcal{W}_{\pi}^{2}$ is the following well-known frequency-domain representation. For every stable LTI system $T: \mathcal{P} \mathcal{W}_{\pi}^{2} \rightarrow \mathcal{P} \mathcal{W}_{\pi}^{2}$ there exists exactly one function $\hat{h}_{T} \in L^{\infty}[-\pi, \pi]$ such that

$$
(T f)(t)=\frac{1}{2 \pi} \int_{-\pi}^{\pi} \hat{f}(\omega) \hat{h}_{T}(\omega) \mathrm{e}^{i \omega t} \mathrm{~d} \omega
$$

for all $f \in \mathcal{P W}_{\pi}^{2}$, and the operator norm of $T$ is given by $\|T\|=$ $\left\|\hat{h}_{T}\right\|_{\infty}$. Conversely, every function $\hat{h}_{T} \in L^{\infty}[-\pi, \pi]$ defines a stable LTI system $T: \mathcal{P} \mathcal{W}_{\pi}^{2} \rightarrow \mathcal{P} \mathcal{W}_{\pi}^{2}$. Thus, the space of all stable LTI systems defined on $\mathcal{P} \mathcal{W}_{\pi}^{2}$ is isometrically isomorphic to $L^{\infty}[-\pi, \pi]$. Note that $\hat{h}_{T} \in L^{\infty}[-\pi, \pi] \subset L^{2}[-\pi, \pi]$ and consequently $h_{T} \in \mathcal{P} \mathcal{W}_{\pi}^{2}$. Another possible representation for stable LTI systems operating on signals in $\mathcal{P W}_{\pi}^{2}$ is the following time-domain representation in the form of a convolution integral. For every stable LTI system $T: \mathcal{P} \mathcal{W}_{\pi}^{2} \rightarrow \mathcal{P} \mathcal{W}_{\pi}^{2}$, we have

$$
(T f)(t)=\int_{-\infty}^{\infty} f(\tau) h_{T}(t-\tau) \mathrm{d} \tau
$$

for all $f \in \mathcal{P W}_{\pi}^{2}$, where $h_{T}=T$ sinc $\in L^{2}(\mathbb{R})$. However, for stable LTI systems operating on other signal spaces, such a convolution integral representation does not necessarily exist. For example, it has been shown that there exist stable LTI systems $T: \mathcal{P} \mathcal{W}_{\pi}^{1} \rightarrow \mathcal{P} \mathcal{W}_{\pi}^{1}$, which do not have a convolution integral representation in the form of (3), because the integral diverges for some signal $f \in \mathcal{P} \mathcal{W}_{\pi}^{1}$ [20]. In contrast, the frequency domain representation (2) is possible for all stable LTI systems $T: \mathcal{P W}_{\pi}^{1} \rightarrow \mathcal{P W}_{\pi}^{1}$.
Before we continue the discussion, we introduce the Hilbert transform and the ideal low-pass filter, which will be needed subsequently and which serve as illustrative examples of two stable LTI systems. Both systems are important in theoretical analyses [23], [22]. Although it is not possible to realize them exactly in practice, they can be seen as the limit case of realizable systems.

The Hilbert transform $H f$ of a signal $f$ is defined by

$$
(H f)(t)=\frac{1}{2 \pi} \int_{-\infty}^{\infty}(-i \operatorname{sgn}(\omega)) \hat{f}(\omega) \mathrm{e}^{i \omega t} \mathrm{~d} \omega
$$

where sgn denotes the signum function. It is well known that the Hilbert transform $H: \mathcal{P W}_{\pi}^{2} \rightarrow \mathcal{P W}_{\pi}^{2}$ is an isometric isomorphism. Hence, $H$ is a stable LTI system with $\|H\|=$ $\sup _{\|f\|_{\mathcal{P} \mathcal{W}^{2}} \leq 1}\|H f\|_{\mathcal{P} \mathcal{W}_{\pi}^{2}}=1$ that maps $\mathcal{P} \mathcal{W}_{\pi}^{2}$ onto $\mathcal{P} \mathcal{W}_{\pi}^{2}$.

The Hilbert transform has many applications [23], [22]. One is in communication theory where the Hilbert transform is used to define the analytical signal

$$
f_{+}=f+i H f \text {. }
$$

By $S_{+}$we denote the analytical signal operator that maps $f \mapsto$ $f_{+}$according to (4). One of the key properties of the analytical signal is the fact that its Fourier transform $\hat{f}_{+}$is zero for negative frequencies. For $f \in \mathcal{P} \mathcal{W}_{\pi}^{2}$ the analytical signal $f_{+}$is well defined, and we have

$$
\left\|f_{+}\right\|_{\mathcal{P} \mathcal{W}_{\pi}^{2}} \leq\|f\|_{\mathcal{P} \mathcal{W}_{\pi}^{2}}+\|H f\|_{\mathcal{P} \mathcal{W}_{\pi}^{2}}=2\|f\|_{\mathcal{P} \mathcal{W}_{\pi}^{2}} .
$$

In Section V, we will see that the computation of the analytical signal is problematic for the space $\mathcal{Z}_{\pi}$.

The ideal low-pass filter $L_{\omega_{g}}: \mathcal{P} \mathcal{W}_{\pi}^{2} \rightarrow \mathcal{P} \mathcal{W}_{\omega_{g}}^{2}$ with bandwidth $0<\omega_{g} \leq \pi$ is defined by

$$
\left(L_{\omega_{g}} f\right)(t)=\frac{1}{2 \pi} \int_{-\omega_{g}}^{\omega_{g}} \hat{f}(\omega) \mathrm{e}^{i \omega t} \mathrm{~d} \omega .
$$

Obviously, $L_{\omega_{g}}$ is, like the Hilbert transform, a stable LTI system with $\left\|L_{\omega_{g}}\right\|=\sup _{\|f\|_{\mathcal{P} \mathcal{W}_{\pi}^{2}} \leq 1}\left\|L_{\omega_{g}} f\right\|_{\mathcal{P} \mathcal{W}_{\pi}^{2}}=1$.

\section{MOTIVATION}

In this paper, we consider a convolution-type system representation. As in the case of sampling series, the theory for stable LTI systems operating on bandlimited signals with finite energy is simple. It is well known [26], [27] that every stable LTI system $T: \mathcal{P} \mathcal{W}_{\pi}^{2} \rightarrow \mathcal{P} \mathcal{W}_{\pi}^{2}$ has the representation

$$
(T f)(t)=\int_{-\infty}^{\infty} f(\tau) h_{T}(t-\tau) \mathrm{d} \tau
$$

with

$$
h_{T}=T \text { sinc } \in \mathcal{P} \mathcal{W}_{\pi}^{2}
$$

where

$$
\operatorname{sinc}(t)= \begin{cases}\frac{\sin (\pi t)}{\pi t}, & t \neq 0 \\ 1, & t=0\end{cases}
$$


Example 1: For the ideal low-pass filter $L_{\omega_{g}}$ with bandwidth $0<\omega_{g} \leq \pi$ and the Hilbert transform $H$, we have

$$
h_{L_{\omega_{g}}}(t)=\left(L_{\omega_{g}} \operatorname{sinc}\right)(t)=\frac{\sin \left(\omega_{g} t\right)}{\pi t}
$$

and

$$
h_{H}(t)=(H \operatorname{sinc})(t)=\frac{\sin ^{2}\left(\frac{\pi}{2} t\right)}{\frac{\pi}{2} t} .
$$

In [19], Habib establishes the convolution representation (5) for signals $f$ from the Zakai space $\mathcal{Z}_{\pi}$ and certain stable linear systems $T$. If the integral is taken as an extended Riemann integral, he proves that (5) holds for all $f \in \mathcal{Z}_{\pi}$ and $t \in \mathbb{R}$ under the following assumptions on the linear system $T$ :

i) $T$ maps $\mathcal{Z}_{\pi}$ into $\mathcal{Z}_{\pi}$ and is bounded with respect to these spaces;

ii) $T$ maps $\mathcal{P} \mathcal{W}_{\pi}^{2}$ into $\mathcal{P} \mathcal{W}_{\pi}^{2}$.

This is an interesting generalization of the results in [21]. However, in [19], no examples of systems that fulfill the assumptions i) and ii) are given. In this paper, we analyze the convergence behavior of the convolution integral

$$
\int_{-\infty}^{\infty} f(\tau) h_{T}(t-\tau) \mathrm{d} \tau
$$

for the ideal low-pass filter $T=L_{\omega_{q}}$ with bandwidth $\omega_{g}$ less than or equal to the signal bandwidth and Hilbert transform $T=H$, and signals $f \in \mathcal{Z}_{\pi}$. In the next section we will show that there exist signals $f \in \mathcal{Z}_{\pi}$ such that (6) diverges for all $t \in \mathbb{R}$ if $T$ is one of the above systems. Consequently, the class of permissible systems in [19] is strongly restricted. It does not include the ideal low-pass filter with bandwidth less than or equal to the signal bandwidth and the Hilbert transform.

\section{DiVERGENCE RESUlT FOR Signals FROM THE ZAKAI CLASS}

The following result shows that the convolution integral (6) diverges for certain signals $f \in \mathcal{Z}_{\pi}$ if $T$ is the ideal low-pass filter with bandwidth less than or equal to the signal bandwidth or the Hilbert transform.

Theorem 1: Let $0<\omega_{g} \leq \pi$ and $a>0$. Then there exist a signal $f_{1} \in \mathcal{Z}_{\pi}$ such that for all $t \in \mathbb{R}$

$$
\limsup _{N \rightarrow \infty}\left|\int_{-N}^{N} f_{1}(\tau) \frac{\sin \left(\omega_{g}(t-\tau)\right)}{\pi(t-\tau)} \mathrm{d} \tau\right|=\infty
$$

and a signal $f_{2} \in \mathcal{Z}_{\pi}$ such that for all $t \in \mathbb{R}$

$$
\limsup _{N \rightarrow \infty}\left|\int_{-N}^{N} f_{2}(\tau) \frac{\sin ^{2}\left(\frac{a \pi}{2}(t-\tau)\right)}{\frac{\pi}{2}(t-\tau)} \mathrm{d} \tau\right|=\infty .
$$

Remark 2: Theorem 1 states the existence of a signal $f_{1} \in \mathcal{Z}_{\pi}$ such that the convolution integral in (7) diverges. In the proof of Theorem 1 we do not only prove the existence, but also give an explicit construction of the signal $f_{1}$.
Remark 3: In Section V, we will use Theorem 1 to derive Corollary 1, which states that the Hilbert transform is an unbounded operator for the space $\mathcal{Z}_{\pi}$.

For the proof of Theorem 1 we need Lemma 1.

Lemma 1: For all $0<\alpha<1 / 2$ and $0 \leq \omega_{g} \leq \pi$, we have

$$
\limsup _{N \rightarrow \infty} \int_{-\pi}^{\pi} \frac{1}{\left|\omega-\omega_{g}\right|^{\alpha}} \frac{\sin \left(N\left(\omega-\omega_{g}\right)\right)}{\omega-\omega_{g}} \mathrm{~d} \omega=\infty .
$$

Proof of Lemma 1: Let $0<\alpha<1 / 2$ and $0 \leq \omega_{g} \leq \pi$ be arbitrary but fixed. For $\lambda \in \mathbb{R}$, we denote by $\lfloor\lambda\rfloor$ the greatest integer that is smaller or equal to $\lambda$. Then we have

$$
\begin{aligned}
\mid \int_{-\pi}^{\pi} & \frac{1}{\left|\omega-\omega_{g}\right|^{\alpha}} \frac{\sin \left(\lambda\left(\omega-\omega_{g}\right)\right)}{\omega-\omega_{g}} \mathrm{~d} \omega \\
& -\int_{-\pi}^{\pi} \frac{1}{\left|\omega-\omega_{g}\right|^{\alpha}} \frac{\sin \left(\lfloor\lambda\rfloor\left(\omega-\omega_{g}\right)\right)}{\omega-\omega_{g}} \mathrm{~d} \omega \mid \\
= & \mid \int_{-\pi}^{\pi} \frac{1}{\left|\omega-\omega_{g}\right|^{\alpha}} \frac{2 \sin \left(\frac{\lambda-\lfloor\lambda\rfloor}{2}\left(\omega-\omega_{g}\right)\right)}{\omega-\omega_{g}} \\
& \times \cos \left(\frac{(\lambda+\lfloor\lambda\rfloor)\left(\omega-\omega_{g}\right)}{2}\right) \mathrm{d} \omega \mid \\
\leq & \int_{-\pi}^{\pi} \frac{1}{\left|\omega-\omega_{g}\right|^{\alpha}} \mathrm{d} \omega \\
\leq & 6 \pi .
\end{aligned}
$$

Moreover, for $T>1$, we obtain

$$
\begin{aligned}
\frac{1}{T} & \int_{0}^{T} \int_{-\pi}^{\pi} \frac{1}{\left|\omega-\omega_{g}\right|^{\alpha}} \frac{\sin \left(\lambda\left(\omega-\omega_{g}\right)\right)}{\omega-\omega_{g}} \mathrm{~d} \omega \mathrm{d} \lambda \\
& =\int_{-\pi}^{\pi} \frac{1}{\left|\omega-\omega_{g}\right|^{\alpha}}\left(\frac{1}{T} \int_{0}^{T} \frac{\sin \left(\lambda\left(\omega-\omega_{g}\right)\right)}{\omega-\omega_{g}} \mathrm{~d} \lambda\right) \mathrm{d} \omega \\
& =\int_{-\pi}^{\pi} \frac{1}{\left|\omega-\omega_{g}\right|^{\alpha}} \frac{2 \sin ^{2}\left(\frac{T}{2}\left(\omega-\omega_{g}\right)\right)}{T\left(\omega-\omega_{g}\right)^{2}} \mathrm{~d} \omega \\
& =\int_{-T\left(\pi+\omega_{g}\right) / 2}^{T\left(\pi-\omega_{g}\right) / 2}\left(\frac{T}{2}\right)^{\alpha} \frac{\sin ^{2}(\xi)}{|\xi|^{\alpha+2}} \mathrm{~d} \xi \\
& \geq\left(\frac{T}{2}\right)^{\alpha} \int_{-\pi / 2}^{0} \frac{\sin ^{2}(\xi)}{|\xi|^{\alpha+2}} \mathrm{~d} \xi \\
& \geq\left(\frac{T}{2}\right)^{\alpha}\left(\frac{2}{\pi}\right)^{\alpha+2} \frac{\pi}{4}
\end{aligned}
$$

and consequently

$$
\limsup _{\lambda \rightarrow \infty} \int_{-\pi}^{\pi} \frac{1}{\left|\omega-\omega_{g}\right|^{\alpha}} \frac{\sin \left(\lambda\left(\omega-\omega_{g}\right)\right)}{\omega-\omega_{g}} \mathrm{~d} \omega=\infty .
$$

Combining (10) and (11) gives the assertion (9).

Now we are in the position to prove Theorem 1.

Proof of Theorem 1: We start with the proof of (7). Let $0<$ $\omega_{g} \leq \pi$ and $0<\alpha<1 / 2$ be arbitrary but fixed. For

$$
G(\omega)= \begin{cases}\left|\omega-\omega_{g}\right|^{-\alpha}, & \text { if }-\pi \leq \omega \leq \pi \\ 0, & \text { otherwise }\end{cases}
$$


consider the signal

$$
g(t)=\frac{1}{2 \pi} \int_{-\pi}^{\pi} G(\omega) \mathrm{e}^{i \omega t} \mathrm{~d} \omega .
$$

Since $G \in L^{2}(\mathbb{R}) \cap L^{1}(\mathbb{R})$ it follows that $g \in \mathcal{P} \mathcal{W}_{\pi}^{2}$ and that $g$ is continuous and bounded on $\mathbb{R}$. Furthermore, let $f(t)=t g(t)$. Since $g \in \mathcal{P} \mathcal{W}_{\pi}^{2}$ we have $|g(z)| \leq\|g\|_{\mathcal{P} \mathcal{W}_{\pi}^{2}} \mathrm{e}^{\pi|\operatorname{Im}(z)|}, z \in \mathbb{C}$, and consequently $|f(z)| \leq|z|\|g\|_{\mathcal{P W}_{\pi}^{2}} \mathrm{e}^{\pi|\operatorname{Im}(z)|}$. Thus, the Paley-Wiener-Schwartz theorem implies that $\hat{f}$ is a distribution with support in $[-\pi, \pi]$. Moreover, we have $\|f\|_{\mathcal{Z}_{\pi}}=\int_{-\infty}^{\infty}|t g(t)|^{2} /\left(1+t^{2}\right) \mathrm{d} t \leq 2 \pi\|g\|_{\mathcal{P W}_{\pi}^{2}}^{2}<\infty$. Thus, $f$ is a signal in $\mathcal{Z}_{\pi}$.

In the following, let $t \in \mathbb{R}$ be arbitrary but fixed. We have

$$
\begin{aligned}
& \left|\int_{-N}^{N} f(\tau) \frac{\sin \left(\omega_{g}(t-\tau)\right)}{\pi(t-\tau)} \mathrm{d} \tau\right| \\
& =\left|\int_{-N}^{N}(t g(\tau)-(t-\tau) g(\tau)) \frac{\sin \left(\omega_{g}(t-\tau)\right)}{\pi(t-\tau)} \mathrm{d} \tau\right| \\
& \quad \geq\left|\int_{-N}^{N} g(\tau) \frac{\sin \left(\omega_{g}(t-\tau)\right)}{\pi} \mathrm{d} \tau\right| \\
& \quad-\left|t \int_{-N}^{N} g(\tau) \frac{\sin \left(\omega_{g}(t-\tau)\right)}{\pi(t-\tau)} \mathrm{d} \tau\right| .
\end{aligned}
$$

Since

$$
\begin{aligned}
&\left|\int_{-N}^{N} g(\tau) \frac{\sin \left(\omega_{g}(t-\tau)\right)}{\pi} \mathrm{d} \tau\right| \\
& \geq \frac{1}{2 \pi}\left|\int_{-N}^{N} g(\tau) \mathrm{e}^{-i \omega_{g} \tau} \mathrm{d} \tau\right| \\
&-\frac{1}{2 \pi}\left|\int_{-N}^{N} g(\tau) \mathrm{e}^{i \omega_{g} \tau} \mathrm{d} \tau\right| \\
&= \frac{1}{(2 \pi)^{2}}\left|\int_{-\pi}^{\pi} G(\omega) \int_{-N}^{N} \mathrm{e}^{i\left(\omega-\omega_{g}\right) \tau} \mathrm{d} \tau \mathrm{d} \omega\right| \\
&-\frac{1}{2 \pi}\left|\int_{-N}^{N} g(\tau) \mathrm{e}^{i \omega_{g} \tau} \mathrm{d} \tau\right| \\
&= \frac{1}{2 \pi^{2}}\left|\int_{-\pi}^{\pi} G(\omega) \frac{\sin \left(N\left(\omega-\omega_{g}\right)\right)}{\omega-\omega_{g}} \mathrm{~d} \omega\right| \\
&-\frac{1}{2 \pi}\left|\int_{-N}^{N} g(\tau) \mathrm{e}^{i \omega_{g} \tau} \mathrm{d} \tau\right|
\end{aligned}
$$

where the interchange of the integrations with respect to $\tau$ and $\omega$ is possible because $G$ is absolutely integrable, we obtain

$$
\begin{aligned}
& \left|\int_{-N}^{N} f(\tau) \frac{\sin \left(\omega_{g}(t-\tau)\right)}{\pi(t-\tau)} \mathrm{d} \tau\right| \\
& \geq \frac{1}{2 \pi^{2}}\left|\int_{-\pi}^{\pi} G(\omega) \frac{\sin \left(N\left(\omega-\omega_{g}\right)\right)}{\omega-\omega_{g}} \mathrm{~d} \omega\right| \\
& \quad-\frac{1}{2 \pi}\left|\int_{-N}^{N} g(\tau) \mathrm{e}^{i \omega_{g} \tau} \mathrm{d} \tau\right| \\
& \quad-\left|t \int_{-N}^{N} g(\tau) \frac{\sin \left(\omega_{g}(t-\tau)\right)}{\pi(t-\tau)} \mathrm{d} \tau\right| .
\end{aligned}
$$

Moreover, we have

$$
\begin{aligned}
\lim _{N \rightarrow \infty}\left|t \int_{-N}^{N} g(\tau) \frac{\sin \left(\omega_{g}(t-\tau)\right)}{\pi(t-\tau)} \mathrm{d} \tau\right| \\
=\left|t\left(L_{\omega_{g}} g\right)(t)\right| \leq|t|\|g\|_{\mathcal{P W}_{\pi}^{2}}<\infty
\end{aligned}
$$

because $g \in \mathcal{P} \mathcal{W}_{\pi}^{2}$, and

$$
\begin{aligned}
\lim _{N \rightarrow \infty} \int_{-N}^{N} g(\tau) \mathrm{e}^{i \omega_{g} \tau} \mathrm{d} \tau \\
\quad=\frac{1}{2}\left(\lim _{\omega \downarrow-\omega_{g}} G(\omega)+\lim _{\omega \uparrow-\omega_{g}} G(\omega)\right)<\infty
\end{aligned}
$$

by the Fourier inversion theorem, since $G$ is of bounded variation in a neighborhood of $-\omega_{g}$ [28]. Further, Lemma 1 states that

$$
\limsup _{N \rightarrow \infty}\left|\int_{-\pi}^{\pi} G(\omega) \frac{\sin \left(N\left(\omega-\omega_{g}\right)\right)}{\omega-\omega_{g}} \mathrm{~d} \omega\right|=\infty .
$$

Finally, combining (12)-(15) yields

$$
\limsup _{N \rightarrow \infty}\left|\int_{-N}^{N} f(\tau) \frac{\sin \left(\omega_{g}(t-\tau)\right)}{\pi(t-\tau)} \mathrm{d} \tau\right|=\infty
$$

which is true for all $t \in \mathbb{R}$ because $t$ was arbitrary. We see that $f$ is the desired divergence creating signal $f_{1}$. This completes the proof of (7).

The second part of the Theorem, i.e., (8) can be proven similarly to the first part if the function $g(t)=$ $1 /(2 \pi) \int_{-\pi}^{\pi} G(\omega) \mathrm{e}^{i \omega t} \mathrm{~d} \omega$ with

$$
G(\omega)= \begin{cases}|\omega|^{-\alpha}, & \text { if }-\pi \leq \omega \leq \pi \\ 0, & \text { otherwise }\end{cases}
$$

is used. In this case, the divergence creating signal is given by $f_{2}(t)=t g(t)$.

\section{Applications}

We have shown that for all $t \in \mathbb{R}$ there are signals in $\mathcal{Z}_{\pi}$ such that the convolution integral (6) diverges if $T$ is the Hilbert transform or the ideal low-pass filter with bandwidth less than or equal to the signal bandwidth. Based on this result we can draw some further conclusions.

Next, we use Theorem 1 in conjunction with Theorem 3.1 from [19] to show that the ideal low-pass filter $L_{\omega_{g}}$ with bandwidth $\omega_{g}$ less than or equal to the signal bandwidth and the Hilbert transform $H$ are not bounded operators that map $\mathcal{Z}_{\pi}$ in $\mathcal{Z}_{\pi}$. For the further discussion we use the Hilbert transform. Nevertheless, the same is true for the ideal low-pass filter $L_{\omega_{q}}$.

To see that $H$ is not a bounded operator that maps $\mathcal{Z}_{\pi}$ into $\mathcal{Z}_{\pi}$, we use an indirect proof. Assume $H$ is a bounded operator that maps $\mathcal{Z}_{\pi}$ into $\mathcal{Z}_{\pi}$. Then it follows that $H f$ is in $\mathcal{Z}_{\pi}$. This implies that

$$
|(H f)(t)|<\infty \text { for all } t \in \mathbb{R} .
$$


Furthermore, all assumption of Theorem 3.1 in [19] are fulfilled. According to this theorem, we have

$$
\begin{aligned}
(H f)(t) & =\lim _{N \rightarrow \infty} \int_{-N}^{N} f(\tau)\left(H \frac{\sin (a \pi(\cdot-\tau))}{\pi(\cdot-\tau)}\right)(t) \mathrm{d} \tau \\
& =\lim _{N \rightarrow \infty} \int_{-N}^{N} f(\tau) \frac{\sin ^{2}\left(\frac{a \pi}{2}(\tau-t)\right)}{\frac{\pi}{2}(\tau-t)} \mathrm{d} \tau
\end{aligned}
$$

for all $f \in \mathcal{Z}_{\pi}$, all $a>1$, and all $t \in \mathbb{R}$. However, in Theorem 1 , we have seen that for every $t \in \mathbb{R}$ and $a>0$ there exists a signal $f_{2} \in \mathcal{Z}_{\pi}$ such that

$$
\limsup _{N \rightarrow \infty} \int_{-N}^{N} f_{2}(\tau) \frac{\sin ^{2}\left(\frac{a \pi}{2}(\tau-t)\right)}{\frac{\pi}{2}(\tau-t)} \mathrm{d} \tau=\infty .
$$

This is a contradiction to (16). Therefore, it follows that $H$ is not a bounded operator that maps $\mathcal{Z}_{\pi}$ into $\mathcal{Z}_{\pi}$. This implies that the Hilbert transform $H$ is not included in the class of systems for which Theorem 3.1 in [19] is valid.

Next, we consider the space $\mathcal{P} \mathcal{W}_{\pi}^{2}$ which is dense in $\mathcal{Z}_{\pi}$, equipped with the norm $\|\cdot\|_{\mathcal{Z}_{\pi}}$. For this subspace, the Hilbert transform is well defined, and we have $H f \in \mathcal{Z}_{\pi}$ for all $f \in \mathcal{P} \mathcal{W}_{\pi}^{2}$. However, as shown by the following corollary, the Hilbert transform $H:\left(\mathcal{P} \mathcal{W}_{\pi}^{2},\|\cdot\|_{\mathcal{Z}_{\pi}}\right) \rightarrow \mathcal{Z}_{\pi}$, taken as an operator that maps the subspace $\mathcal{P} \mathcal{W}_{\pi}^{2}$ of $\mathcal{Z}_{\pi}$ into $\mathcal{Z}_{\pi}$, is an unbounded operator.

Corollary 1: The Hilbert transform $H:\left(\mathcal{P W} \mathcal{W}_{\pi}^{2},\|\cdot\|_{\mathcal{Z}_{\pi}}\right) \rightarrow$ $\mathcal{Z}_{\pi}$ and the analytical signal operator $S_{+}:\left(\mathcal{P} \mathcal{W}_{\pi}^{2},\|\cdot\|_{\mathcal{Z}_{\pi}}\right) \rightarrow$ $\mathcal{Z}_{\pi}$ are unbounded and, therefore, discontinuous operators.

Proof: The unboundedness of the Hilbert transform $H$ : $\left(\mathcal{P W} \mathcal{W}_{\pi}^{2},\|\cdot\|_{\mathcal{Z}_{\pi}}\right) \rightarrow \mathcal{Z}_{\pi}$ can be easily seen, because the converse assumption leads to a contradiction. Suppose $H:\left(\mathcal{P} \mathcal{W}_{\pi}^{2},\|\cdot\|_{\mathcal{Z}_{\pi}}\right)$ $\rightarrow \mathcal{Z}_{\pi}$ is a bounded operator. Then it is possible to extend this densely defined operator to a bounded operator $H: \mathcal{Z}_{\pi} \rightarrow \mathcal{Z}_{\pi}$. But we have already proven that $H$ is not a bounded operator that maps $\mathcal{Z}_{\pi}$ in $\mathcal{Z}_{\pi}$. This leads to a contradiction. Therefore, it follows that $H:\left(\mathcal{P} \mathcal{W}_{\pi}^{2},\|\cdot\|_{\mathcal{Z}_{\pi}}\right) \rightarrow \mathcal{Z}_{\pi}$ is an unbounded operator, that is

$$
\sup _{\substack{f \in \mathcal{P} \mathcal{W}_{\pi}^{2} \\\|f\|_{\mathcal{Z}_{\pi} \leq 1}}}\|H f\|_{\mathcal{Z}_{\pi}}=\infty
$$

Since $\left\|f_{+}\right\|{\mathcal{\mathcal { Z } _ { \pi }}} \geq\|H f\|_{\mathcal{Z}_{\pi}}-\|f\|_{\mathcal{Z}_{\pi}}$, it follows immediately from (17) that the analytic signal operator $S_{+}:\left(\mathcal{P W} \mathcal{W}_{\pi}^{2},\|\cdot\|_{\mathcal{Z}_{\pi}}\right)$ $\rightarrow \mathcal{Z}_{\pi}$ is not bounded.

The above discussion shows that the computation of the analytic signal is problematic for the space $\mathcal{Z}_{\pi}$. Thus, the results in this paper imply that the concept of the analytical signal, which is extensively used in communications, cannot be generalized to the signal space $\mathcal{Z}_{\pi}$ in general.

\section{ACKNOWLEDGMENT}

The authors would like to thank all the reviewers for giving helpful comments. In particular, they would like to thank the reviewer who provided a simplified version of the proof of Theorem 1.

\section{REFERENCES}

[1] A. J. Jerri, "The Shannon sampling theorem-Its various extensions and applications: A tutorial review," Proc. IEEE, vol. 65, no. 11, pp. 1565-1596, Nov. 1977.

[2] Advanced Topics in Shannon Sampling and Interpolation Theory, R. J. Marks II, Ed.. New York: Springer-Verlag, 1993.

[3] J. R. Higgins, Sampling Theory in Fourier and Signal Analysis—Foundations. Oxford, U.K.: Oxford Univ. Press, 1996.

[4] C. E. Shannon, "Communication in the presence of noise," Proc. IRE, vol. 37, no. 1, pp. 10-21, Jan. 1949

[5] R. P. Gosselin, "On the $L^{p}$ theory of cardinal series," Ann. Math., vol. 78, no. 3, pp. 567-581, Nov. 1963.

[6] M. Zakai, "Band-limited functions and the sampling theorem," Inf. Control, vol. 8, no. 2, pp. 143-158, 1965.

[7] E. Pfaffelhuber, "Sampling series for band-limited generalized functions," IEEE Tran. Inf. Theory, vol. 17, no. 6, pp. 650-654, Nov. 1971.

[8] L. L. Campbell, "Sampling theorem for the Fourier transform of a distribution with bounded support," SIAM J. Appl. Math., vol. 16, no. 3, pp. 626-636, May 1968.

[9] W. Splettstößer, R. Stens, and G. Wilmes, "On approximation by the interpolating series of G. Valiron," Functiones et Approximatio, Commentarii Mathematici, vol. 11, pp. 39-56, 1981.

[10] S. Cambanis and E. Masry, "Truncation error bounds for the cardinal sampling expansion of band-limited signals," IEEE Trans. Inf. Theory, vol. IT-28, no. 4, pp. 605-612, Jul. 1982.

[11] G. Hinsen, "Irregular sampling of bandlimited $L^{p}$-functions," $J . A p$ prox. Theory, vol. 72, no. 3, pp. 346-364, Mar. 1993.

[12] P. J. S. G. Ferreira, "Nonuniform sampling of nonbandlimited signals," IEEE Signal Process. Lett., vol. 2, no. 5, pp. 89-91, May 1995.

[13] A. Aldroubi and K. Gröchenig, "Nonuniform sampling and reconstruction in shift-invariant spaces," SIAM Rev., vol. 43, no. 4, pp. 585-620, Dec. 2001.

[14] Y. Lyubarskii and W. R. Madych, "Interpolation of functions from generalized Paley-Wiener spaces," J. Approx. Theory, vol. 133, no. 2, pp. 251-268, Apr. 2005.

[15] S. Cambanis and E. Masry, "Zakai's class of bandlimited functions and processes: Its characterization and properties," SIAM J. Appl. Math., vol. 30, no. 1, pp. 10-21, 1976.

[16] K. Gröchenig, "Weight functions in time-frequency analysis," in Pseudodifferential Operators: Partial Differential Equations and Time-Frequency Analysis, L. Rodino, Ed. et al. Fields Institute Communications, 2007, vol. 52, pp. 343-366.

[17] A. J. Lee, "On band limited stochastic processes," SIAM J. Appl. Math., vol. 30, no. 2, pp. 269-277, Mar. 1976.

[18] R. L. Stens, "A unified approach to sampling theorems for derivatives and Hilbert transforms," Signal Process., vol. 5, pp. 139-151, 1983.

[19] M. K. Habib, "Digital representations of operators on band-limited random signals," IEEE Trans. Inf. Theory, vol. 47, no. 1, pp. 173-177, Jan. 2001.

[20] H. Boche and U. J. Mönich, "Time domain representation of systems on bandlimited signals," in Proc. IEEE Information Theory Workshop, May 2008, pp. 51-55.

[21] D. H. Mugler, "Convolution, differential equations, and entire functions of exponential type," Trans. Amer. Math. Soc., vol. 216, pp. 145-187, Feb. 1976.

[22] B. F. Logan, Jr., "Theory of analytic modulation systems," Bell Syst Tech. J., vol. 57, no. 3, pp. 491-576, Mar. 1978.

[23] D. Gabor, "Theory of communication," J. Inst. Elect. Eng., vol. 93, no. 3, pp. 429-457, Nov. 1946.

[24] J. Segal, Le Zéro et le Un 2003, Éditions Syllepse.

[25] L. Hörmander, The Analysis of Linear Partial Differential Operators I: Distribution Theory and Fourier Analysis. New York: SpringerVerlag, 1983.

[26] L. Franks, Signal Theory. Upper Saddle River, NJ: Prentice-Hall, 1969.

[27] M. Schetzen, Linear Time-Invariant Systems. New York: Wiley, 2002.

[28] K. Chandrasekharan, Classical Fourier Transforms. New York: Springer-Verlag, 1989. 


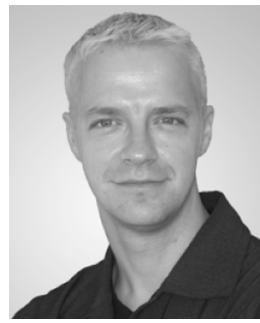

Holger Boche (M'04-SM'07) received the Dipl.-Ing. and Dr.-Ing. degrees in electrical engineering from the Technische Universität Dresden, Germany, in 1990 and 1994, respectively. In 1992, he graduated in mathematics from the Technische Universität Dresden, and in 1998, he received the Dr.rer.nat. degree in pure mathematics from the Technische Universität Berlin, Germany.

From 1994 to 1997, he did postgraduate studies in mathematics at the Friedrich-Schiller Universität Jena, Germany. In 1997, he joined the Heinrich-Hertz-Institut (HHI) für Nachrichtentechnik Berlin. Since 2002, he has been a Full Professor for Mobile Communication Networks at the Technische Universität Berlin at the Institute for Communications Systems. In 2003, he became director of the Fraunhofer German-Sino Lab for Mobile Communications, Berlin, and since 2004, he has also been Director of the Fraunhofer Institute for Telecommunications (HHI), Berlin. He was visiting professor at the ETH Zurich during the winter terms of 2004 and 2006 and at KTH Stockholm during the summer term of 2005.

Prof. Boche received the Research Award "Technische Kommunikation" from the Alcatel SEL Foundation in October 2003, the "Innovation Award" from the Vodafone Foundation in June 2006 and the Gottfried Wilhelm Leibniz Prize from the Deutsche Forschungsgemeinschaft (German Research Foundation) in 2008. He was co-recipient of the 2006 IEEE Signal Processing Society Best Paper Award and recipient of the 2007 IEEE Signal Processing Society Best Paper Award. He is a member of IEEE Signal Processing Society SPCOM and SPTM Technical Committee. He was elected a member of the German Academy of Sciences (Leopoldina) in 2008.

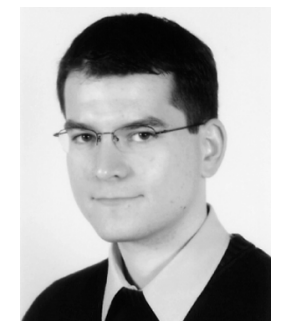

Ullrich J. Mönich (S'06) received the Dipl.-Ing. degree in electrical engineering from the Technische Universität Berlin, Germany, in 2005, where he is currently pursuing the Ph.D. degree.

During the winter term 2003, he was a visiting researcher at the University of California, Santa Barbara. His research activities comprise sampling theory, ultrawideband mobile communications, and information theory. 